Introduction

\title{
Introduction to the 2018 Research Symposium Forum
}

\author{
Swathi Kiran ${ }^{\mathrm{a}}$
}

Purpose: The purpose of this introduction is to provide an overview of the articles contained within this forum of Journal of Speech, Language, and Hearing Research (JSLHR). Each of these articles is based upon presentations from the Research Symposium at the 2018 annual convention of the American Speech-Language-Hearing Association held in Boston, MA.

Question and Answer Panel: https://doi.org/10.23641/ asha. 11120825
A s part of the Research Symposium held at the American Speech-Language-Hearing Association convention every year, presentations that include current advances and innovative ideas on a particular topic are presented. At the 2018 meeting, the topic focused on Advances in Neuroplasticity Research specific to language recovery for people with aphasia. This topic is particularly clinically significant as aphasia affects approximately one third of stroke survivors. According to the National Aphasia Association (2019), about 2 million individuals live with aphasia in the United States, and the numbers are larger across the world. More importantly, difficulty in communication and social isolation that occurs after one suffers from aphasia has a negative impact on one's quality of life, and this impact is greater than other chronic debilitating diseases such as cancer or Alzheimer's disease (Lam \& Wodchis, 2010).

Thus, it is imperative for scientists and clinicians to (a) find what causes and how to prevent a stroke, (b) understand the recovery process, (c) identify biomarkers of recovery, (d) develop and rigorously test different types of interventions for people with aphasia, and (e) facilitate access to effective clinical services that these individuals need. Importantly, recent reviews highlight the advances made in our current understanding of how recovery progresses after a stroke and aphasia (Crosson et al., 2019; Kiran \& Thompson, 2019). The five articles in this forum cover a

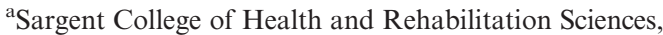

Boston University, MA

Correspondence to Swathi Kiran: kirans@bu.edu

Editor-in-Chief: Sean M. Redmond

Received October 22, 2019

Accepted October 22, 2019

https://doi.org/10.1044/2019_JSLHR-19-00297

Publisher Note: This article is part of the Forum: Advances in

Neuroplasticity Research on Language Recovery in Aphasia. range of topics that emphasize recent advances in understanding neuroplasticity, identifying biomarkers of recovery, and ultimately improving treatment outcomes for aphasia.

In the first article, Turkeltaub (2019) proposes a taxonomy for how behavioral outcomes after a stroke may be related to specific consequences in the brain after a stroke. $\mathrm{He}$ argues that behavioral outcomes after stroke are related to the nature of the stroke size and site, the neurophysiological processes that underlie the sequelae of the stroke, and the resulting recovery process. While all these factors have been known to clinicians and scientists studying language recovery, Turkeltaub proposes a logical breakdown of individual- and process-specific factors that influence recovery.

In the second article, Kristinsson et al. (2019) present some interesting preliminary data on the potential role of genetic markers in characterizing individuals with aphasia in terms of their language severity and potential for recovery. Kristinsson and colleagues found that individuals with aphasia who had the atypical brain-derived neurotrophic factor (BDNF) genotype (individuals with the Val66met or Met66Met alleles) had more severe language impairment than individuals with typical BNDF genotypes. These results are consistent with Turkeltaub's (2019) taxonomy that identifies individual specific features that exist prior to the stroke but may still influence language recovery and outcome.

In the third article, and keeping with the spirit of improving our understanding of language processing, Wilson et al. (2019) describe adaptive language processing paradigms for use in functional magnetic resonance imaging (fMRI) studies. These tasks examine each individual's language processing ability in a dynamic adaptive fashion that can adjust the task level to specific performance criteria. This way, researchers can use a single fMRI task for all their participants but extract individualized performance

Disclosure: The author has declared that no competing interests existed at the time of publication. 
levels as performance varies across participants or changes within participants.

In the fourth and final articles, Thompson (2019) and Kiran, Meier, and Johnson (2019) provide a more in-depth analysis of the mechanisms underlying language recovery after rehabilitation in individuals who present with sentence-processing deficits and lexical-processing deficits, respectively. First, Thompson (2019) describes the rigorously documented efficacy data for the treatment of underlying forms (TUF) approach to treat sentence processing deficits (Thompson \& Shapiro, 2005). She then describes the neural underpinnings of recovered sentenceprocessing skills using a variety of neuroimaging approaches linking behavioral and neuroimaging results back to principles of neuroplasticity in chronic aphasia. In the final article, Kiran et al. (2019) present a provocative mechanistic model of language recovery based on their observations from neuroimaging individuals with aphasia who have word-retrieval deficits and receive rehabilitation for these deficits. They suggest that language recovery involves a bilateral network where spared left hemisphere regions have a dominant and important role and that individuals who present with better brain network properties after stroke are likely to also show greater language recovery. These observations align with the overarching taxonomy presented by Turkeltaub (2019) and with the observation by Kristinsson et al. (2019) that pre-stroke factors such as genetic phenotype and structural and functional connectivity may influence the degree of behavioral recovery after a stroke. More work needs to be done to integrate these interconnected factors that can explain individual differences in language impairment, recovery, and responsiveness to rehabilitation. Only then can we begin to accurately understand and recommend the optimal rehabilitation path for each individual survivor of aphasia.

It is anticipated that these articles will generate a lot of discussion about neuroplasticity, recovery, and rehabilitation among clinicians and researchers engaged in the study of aphasia and will, hopefully, benefit individuals with aphasia by getting them the best clinical care.

\section{Acknowledgments}

This article stems from the 2018 Research Symposium at the American Speech-Language-Hearing Association Convention, which was supported by the National Institute on Deafness and
Other Communication Disorders of the National Institutes of Health under award number R13DC003383. The content is solely the responsibility of the authors and does not necessarily represent the official views of the National Institutes of Health.

\section{References}

Crosson, B., Rodriguez, A. D., Copland, D., Fridriksson, J., Krishnamurthy, L. C., Meinzer, M., ... Leff, A. P. (2019). Neuroplasticity and aphasia treatments: New approaches for an old problem. Journal of Neurology, Neurosurgery \& Psychiatry, 90, 1147-1155. https://doi.org/10.1136/jnnp-2018-319649

Kiran, S., Meier, E. L., \& Johnson, J. (2019). Neuroplasticity in aphasia: A proposed framework of language recovery. Journal of Speech, Language, and Hearing Research, 62, 3973-3985. https://doi.org/10.1044/2019_JSLHR-L-RSNP-19-0054

Kiran, S., \& Thompson, C. K. (2019). Neuroplasticity of language networks in aphasia: Advances, updates and future challenges. Frontiers in Neurology, 10, 295. https://doi.org/10.3389/fneur. 2019.00295

Kristinsson, S., Yourganov, G., Xiao, F., Bonilha, L., Stark, B. C., Rorden, C., ... Fridriksson, J. (2019). Brain-derived neurotrophic factor genotype-specific differences in cortical activation in chronic aphasia. Journal of Speech, Language, and Hearing Research, 62, 3923-3936. https://doi.org/10.1044/ 2019_JSLHR-L-RSNP-19-0021

Lam, J. M., \& Wodchis, W. P. (2010). The relationship of 60 disease diagnoses and 15 conditions to preference-based healthrelated quality of life in Ontario hospital-based long-term care residents. Medical Care, 48(4), 380-387. https://doi.org/10.1097/ MLR.0b013e3181ca2647

National Aphasia Association. (2019). [Homepage]. Retrieved from https://www.aphasia.org/

Thompson, C. K. (2019). Neurocognitive recovery of sentence processing in aphasia. Journal of Speech, Language, and Hearing Research, 62, 3947-3972. https://doi.org/10.1044/2019_JSLHR-LRSNP-19-0219

Thompson, C. K., \& Shapiro, L. P. (2005). Treating agrammatic aphasia within a linguistic framework: Treatment of Underlying Forms. Aphasiology, 19(10-11), 1021-1036. https://doi.org/ $10.1080 / 02687030544000227$

Turkeltaub, P. E. (2019). A taxonomy of brain-behavior relationships after stroke. Journal of Speech, Language, and Hearing Research, 62, 3907-3922. https://doi.org/10.1044/ 2019_JSLHR-L-RSNP-19-0032

Wilson, S. M., Eriksson, D. K., Yen, M., Demarco, A. T., Schneck, S. M., \& Lucanie, J. M. (2019). Language mapping in aphasia. Journal of Speech, Language, and Hearing Research, 62, 3937-3946. https://doi.org/10.1044/2019_JSLHR-L-RSNP19-0031 